1. Skåre C, Calisch TE, Sæter E, Rajka T, Boldingh AM, Nakstad B, Niles DE, Kramer-Johansen J, Olasveengen TM. Implementation and effectiveness of a video-based debriefing programme for neonatal resuscitation. Acta Anaesthesiologica Scandinavica. 2018; 62:394-403. https://doi. org/10.1111/aas.13050.

2. Hurtubise L, Martin B, Gilliland A, Mahan J. To play or not to play: Leveraging video in medical education. Journal of Graduate Medical Education. 2013; 5(1):13-18. http://doi. org/10.4300/JGME-05-01-32.
3. Als, H. Manual for the Naturalistic Observation of Newborn Behavior. NIDCAP Federation International. 2006. Retrieved from http://nidcap.org.

4. Als H, Lester B, Tronick E, Brazelton B. Manual for the Assessment of Preterm Infants' Behavior (APIB). NIDCAP Federation International. 2006. Retrieved from http://nidcap.org.

\title{
Behavioural Language of Preterm Infants: Cardiorespirography and Video Recording of Motor Behaviour as an Integration of the Newborn Indiviualized Developmental Care and Assessment Program (NIDCAP) Observation
}

Ferrari F, Lucaccioni L, Ori L, Talucci G, Cuomo G, Bertoncelli N

Modena University Hospital, Italy

\section{Background}

Understanding early preterm infants' strengths, vulnerabilities, thresholds to stress and disorganization, and behavioural state regulation is of great importance in view of the daily care in the NICU and stabilization of preterm infants. Preterm infants display observable behaviours along three main systems: the autonomic, the motor and the state systems. These behaviours according to the NIDCAP method are coded and recorded on a score sheet based on naturalistic observation, performed with unaided eyes.

\section{Aim}

The primary aim of the study is to check whether cardiorespirography (CRG) and video recording of the motor behaviour may enrich the information derived from the NIDCAP observation.

\section{Method}

We tested a neurophysiological approach to the three NIDCAP observation systems using a cardiorespiratory monitor available in all intensive care units and a time-synchronized video recording of the single motor behaviour of preterm infants. CRG consists of recording heart rate (HR), heart rate variability (HRV), respiration rate, and morphology of the respiration curve and oxygen saturation curve. Time synchronized video recording of the single movement patterns (general movements, startles, tremors, twitches, myoclonic jerks, stretches, trunk arching, isolated arm and leg movements, and eye movements) was performed and compared with the CRG data. From the four main behavioural state parameters (eye movements, body movements, HRV, type and morphology of respiration), we depicted the behavioural state profile. The NIDCAP observation of the infant was also simultaneously performed.

\section{Subjects}

Two hours of CRG and video recording were performed in eight healthy preterm infants from the age of 27 weeks postmenstrual age (PMA) during a NIDCAP observation. Body weight at birth ranged from 635 grams to 900 grams. The mean gestational age at the time of CRG recording was 31.5 weeks PMA, varying from 28 to 34 weeks PMA.

\section{Preliminary results}

Autonomic system: Single isolated acute episodes (short apnea, bradycardia, oxygen desaturation) were present in all eight newborn infants, with various incidence and severity. All had a spontaneous resolution without external intervention. Vagal tone (derived from the HRV curve) seemed to be well differentiated and age-adequate with different amplitude and cycles per minute in active sleep (AS) and quiet sleep (QS) in five infants only.

Motor system: Startle and twitches were seen only in QS whereas general movements, tremors, stretches, myoclonic jerks, trunk arching, and isolated arm and leg movements were predominantly seen in wakefulness and AS. A normal quality of GMs (N GMs) was seen in five infants but a poor repertoire GMs (PR GMs) was observed in another three preterm infants.

State system: The state profile showed a clear behavioural state organization and cyclicity in six infants. In two infants whose PMA was less than 30 weeks, the state organization was hardly recognizable and indeterminate sleep prevailed.

\section{Conclusions}

CRG and synchronized video recording of the spontaneous and interactive behaviour of the preterm infants add extra information to the NIDCAP observation and drives the attention of the staff to those signs of immaturity/instability that deserve further attention and care. 